\title{
Pilot Study on Reducing Symptoms of Anxiety with a Heart Rate Variability Biofeedback Wearable and Remote Stress Management Coach
}

\author{
Adrienne H. Chung ${ }^{1}$ (D) Richard N. Gevirtz ${ }^{2} \cdot$ Raouf S. Gharbo ${ }^{3} \cdot$ Melinda A. Thiam $^{4} \cdot$ J.P. (Jack) Ginsberg ${ }^{5}$
}

Published online: 25 July 2021

(c) The Author(s), under exclusive licence to Springer Science+Business Media, LLC, part of Springer Nature 2021

\begin{abstract}
We assessed the feasibility of using a consumer friendly, heart rate variability biofeedback (HRVB) wearable device in conjunction with a remote stress management coach to reduce symptoms of anxiety. We utilized a discreet, continuously wearable electrocardiogram device, the Lief Smart Patch, which measures and records heart rate and HRV in real time, and guides HRVB exercises using vibrations and visual cues. During the 8 -week study, participants $(N=14)$ wore the Lief Smart Patch, participated in HRVB with the device, utilized the mobile app, and communicated with a remote stress management coach. We collected self-report survey responses to measure symptoms of anxiety (GAD-2) and depression (PHQ-2) every 2 weeks, as well as HRV data throughout the study. Participants' mean GAD-2 score began at 4.6 out of 6 . By the trial's completion, the group's mean GAD-2 score dropped to $1.7\left(\mathrm{t}_{(13)}=11.0, p<.001\right)$ with only 2 of the 14 subjects remaining over the clinical threshold of high anxiety. Similarly, the group's mean PHQ-2 score dropped from 2.93 to $1.29\left(\mathrm{t}_{(13)}=3.54\right.$, $p<.01$ ). In addition, participants increased their HRV (RMSSD) by an average of $+11.4 \mathrm{~ms}$ after participating in a low dose biofeedback exercise. These findings suggest that engaging in HRVB through a discreet wearable device in conjunction with a remote stress management program may be effective for reducing symptoms of anxiety and depression.
\end{abstract}

Keywords Heart rate variability biofeedback · Symptoms of anxiety · Wearable electronic devices · Relaxation training · Autonomic dysfunction

Anxiety disorders are one of the most common mental illnesses in America. The National Institute of Mental Health (2017) estimates that $19.1 \%$ of U.S. adults $(18+)$ have had an anxiety disorder. In addition to affecting over 40 million American adults, anxiety disorders are frequently comorbid with other chronic health conditions (Kroenke et al., 2007). For example, U.S. epidemiological data indicate that 59\% of individuals with generalized anxiety disorder (GAD) also meet the criteria for major depression disorder (MDD), the

Adrienne H. Chung

Adrienne@getlief.com

1 Lief Therapeutics, 2703 Seventh Street \#301, Mailbox \#123, Berkeley, CA 94710, USA

2 Alliant International University, San Diego, USA

3 Virginia Commonwealth University, Richmond, USA

4 Future Psych Solutions, Columbia, USA

5 Saybrook University, Pasadena, USA most severe sub-type of depressive disorder (Carter et al., 2001). As a result, GAD and MDD are one of the most prevalent co-morbidities seen in mental health (Brown et al., 2001; Gao et al., 2013).

The consequences of untreated anxiety and depression have a ripple effect across society and the medical system. The clinical complications of comorbid anxiety and depression include increased risk of suicide, increased risk of psychiatric hospitalization, increased disability, decreased compliance with medical treatments, and significantly higher utilization of medical services (Devane et al., 2005; Hirschfeld, 2001; Katon, 1996; Marcus et al., 1997). In addition, patients with anxiety and depression comorbidity tend to have more chronic and recurrent forms of illness that require long-term treatment (Hirschfeld, 2001). Considering that $90 \%$ of the $\$ 3.8$ trillion the U.S. spends annually on health care is for chronic and mental health conditions (Centers for Disease Control \& Prevention, 2021), the need for effective, affordable mental health solutions is urgent. 
Though highly treatable, only $36.9 \%$ of adults with anxiety (Anxiety \& Depression Association of America, 2020) in the U.S. are receiving care. This contrasts with the statistic that $65 \%$ of adults who have experienced a major depressive episode have received help (National Institute of Mental Health, 2019a, 2019b). Thus, although anxiety impacts over four times more U.S. adults than depression (The National Institute of Mental Health, 2017, 2019a, 2019b), treatment for anxiety disorders lags far behind depression.

Barriers to treatment for anxiety disorders can be summarized into three categories: acceptability, availability, and accessibility. Acceptability of treatment is often hindered by stigma associated with mental illness, lack of perceived efficacy, and discomfort with healthcare professionals. (Jimenez et al., 2013; Mojtabai, 2007; Robinson et al., 2012).

Another challenge for adequate diagnosis and treatment of anxiety disorders is availability and accessibility of mental health care. An estimated 111 million people in the U.S. live in "mental health professional shortage" areas (U.S. Department of Health and Human Services, 2016). The National Council for Behavioral Health (2017) reported that $77 \%$ of U.S. counties are underserved by psychiatrists, which is further exacerbated by the fact that the pool of psychiatrists working with public sector and insured populations declined by 10\% from 2003 to 2013 (Bishop et al., 2016). In addition, the U.S. Department of Health and Human Services (2016) reported that by 2025 , shortages are projected for clinical, counseling, and school psychologists; mental health and substance abuse social workers; school counselors; and marriage and family therapists. The nationwide shortage of behavioral health providers has made it increasingly difficult for many to access critical tools and support to alleviate their symptoms of anxiety.

Worsening matters, the onset of the COVID-19 public health emergency significantly harmed mental health in communities across the globe (Salari et al., 2020). The psychological ramifications of isolation due to quarantine led to increases in anxiety, depression, anger, confusion and stress (Ettman et al., 2020; Salari et al., 2020; Twenge \& Joiner, 2020). However, a silver lining is that the COVID-19 pandemic propelled the adoption of telehealth as a necessary and useful facilitator of high quality remote mental health care (American Psychiatric Association, 2020).

During the pandemic, the U.S. Centers for Medicare \& Medicaid Services significantly expanded the list of covered telehealth services, including those for mental health (U.S. Department of Health \& Human Services, 2020). Furthermore, telehealth coverage was expanded to allow mental health providers equal reimbursement for telehealth visits compared to in-person visits. While this was a significant step forward, the need for mental health support still exceeds the available supply of behavioral health providers, exacerbated by the fact that $40.9 \%$ of U.S. adults reported worsening mental health due to the COVID-19 pandemic (Czeisler et al., 2020).

A growing body of evidence demonstrates that telehealth can effectively facilitate positive mental health outcomes (Firth et al., 2017; Hilty et al., 2013; Mace et al., 2018; Substance Abuse \& Mental Health Services Administration, 2015; Totten et al., 2016). In addition to helping to reduce in person contact during a pandemic, telehealth provides convenient, accessible, affordable, effective care to patients who live in mental health professional shortage areas (Alegría \& O'Malley, 2020). Aligned with the growing use of telehealth and telemedicine for mental health (Barnett \& Huskamp, 2020), wearable devices that help mitigate the somatic symptoms of anxiety are increasingly available to patients and consumers. While other modes of managing the somatic symptoms of anxiety, such as aided meditation, false physiological feedback, electrodermal biofeedback, and respiration biofeedback, are less supported, studies indicate that heart rate variability biofeedback (HRVB) devices can be effective and beneficial for treating anxiety-related symptoms (Hunkin et al., 2019).

Anxiety disorders are often maintained through frequent perceptions of threat or danger (Clark, 1999), which can be exacerbated by low heart rate variability (HRV) (Chalmers et al., 2014). HRV is a measurement of changes in heart rate over time, which provides information about autonomic functioning (Fiskum, 2019). The autonomic nervous system (ANS) is comprised of two systems that send signals to the rest of the body to either stimulate or relax different involuntary processes, like heart rate, blood pressure, breathing, and digestion. The Sympathetic Nervous System (SNS) activates when the body detects danger, which leads to physiological arousal, increased heart rate, increased blood pressure, and increased sensitivity to threats that enables survival if one is confronted with danger. When the body detects the threat is over, the Parasympathetic Nervous System (PNS) activates, so growth and repair can occur, inflammation is reduced, and immunity is improved (Ginsberg \& Nagpal, 2016).

$\mathrm{HRV}$ is considered a measure of variability in our ANSvariability in heart rate reflects adaptability to external triggers (Petrowski et al., 2017). High HRV indicates that the ANS is balanced, which enables the body to respond to many different types of external stimuli with flexibility and resilience. In contrast, low HRV, indicates the ANS is imbalanced. For individuals with anxiety, this often means the sympathetic "fight or flight" nervous system response is operating in overdrive, and prevents the body from activating the PNS.

ANS dysregulation impacts both physical (e.g., increasing cardiovascular risk) and mental (e.g., compromising psychological well-being) health at multiple levels. Loss of regulation of autonomic control of cardiac adjustment to environmental stressors leads to negative impacts on 
physiological function, affecting arterial blood pressure, heart rate and rhythm, and vagal afference (Ginsberg \& Nagpal, 2016). Dysregulation of the autonomic nervous control of the cardiovascular system is associated with increased sympathetic response and reduced parasympathetic tone, and plays an important role in coronary artery disease and the genesis of potentially lethal ventricular arrhythmias (Kim et al., 2018).

A dysfunctional ANS, with an associated reduction in $\mathrm{HRV}$, has been found in a wide range of psychiatric disorders (Young \& Benton, 2018). Anxiety, depression, insomnia, and trauma disorders are thought to be associated with low HRV due to the common thread of dysregulation of the ANS, increased sympathetic arousal, and increased inflammation. Many of these disorders are characterized by decreased vagal tone, which can lead to poor emotion regulation and stress tolerance, and increased social anxiety (Jelinek \& Khanoker, 2020). Echoing these findings, Chalmer et al.'s (2014) meta-analysis of 36 studies found anxiety disorders, such as PTSD, GAD, panic disorder, and social anxiety disorder, are associatively linked with low HRV.

Over the last two decades, there has been growing evidence supporting the use of HRVB to treat a variety of psychiatric disorders and medical conditions, and enhance sports performance (Gevirtz, 2013; Lehrer, 2013). Biofeedback is defined as a technique that enables individuals to learn how to change physiological activity, like HRV, for the purposes of improving health and performance (Gilbert \& Moss, 2003; Schwartz \& Andrasik, 2003; Shaffer \& Moss, 2006). Biofeedback devices provide the wearer information about their physiological processes, enabling them to increase awareness of these processes and be empowered to exert control over their physical and mental state. More specifically, HRVB teaches individuals to strengthen their ANS to improve regulation of the nervous system and improve vagal tone (Lehrer \& Gevirtz, 2014).

HRVB involves the modulation of the ANS through slowed breathing. This is considered a durable skill that induces HRV coherence, which maximizes HRV and facilitates autonomic and cardiorespiratory homeostasis (Burch et al., 2020). HRVB is typically guided by a visual, auditory, or tactile representation of one's heartbeat in real-time in order to increase the amplitude of HRV (Lehrer \& Gevirtz, 2014). During respiration, vagal inhibition initiated in the brainstem allows heart rate to increase, while during exhalation, the vagal brake is initiated leading to heart rate slowing, creating an oscillation in heart rate called respiratory sinus arrhythmia (RSA) that can be accentuated through slowed breathing, especially in resonance frequency. The most established mechanism for HRVB involves establishing autonomic homeostasis through this approach.

HRVB can be used as an effective treatment for increasing HRV and improving mental health treatment outcomes
(Gevirtz, 2013). HRVB is distinct from other standard medical and integrative treatments in that it actively and objectively modulates cardiac vagal tone to 'defuel' physiological arousal (Gharbo, 2020), which can help reduce reactivity to anxiety provoking triggers. A systematic review of HRVB for psychiatric disorders found that it significantly improved clinical symptoms (e.g., anxiety disorders, depression, panic disorder, and PTSD) in $70 \%$ of the studies reviewed (Schoenberg \& David, 2014). Similarly, Lee et al. (2015) pilot study found that a brief HRVB training was related to a significant decrease in trait anxiety.

HRVB can also help improve symptoms of stress, depression, fatigue, pain, and insomnia, which are similarly characterized by SNS over-activation and autonomic imbalance. A 24-study meta-analysis indicated that HRVB resulted in large reductions in self-reported stress and anxiety with effect sizes in the moderate range (Goessl et al., 2017). In addition, Lehrer et al.'s (2020) 58-study metaanalysis found that HRVB had a relatively large effect size on anxiety, compared to other outcomes studied in the context of HRVB, like PTSD and sleep.

Despite the promise of HRVB, there have been limitations to the reach and impact of these techniques due to a lack of portable or wearable devices with high signal quality and effective training protocols. The majority of evidence for HRVB has been derived from studies conducted in a laboratory environment, severely limiting the accessibility and breadth of application of these wellnesspromoting techniques. By not restricting access to HRVB and mindfulness-based stress management practices to clinical environments (Cavanagh et al., 2013; Henriques et al., 2011; Jazaieri et al., 2012), emerging HRVB wearable devices have the potential to remotely extend clinical care teams, and ensure that patients can receive help no matter where they live.

Furthermore, patients with anxiety who feel apprehensive about meeting a psychologist or psychiatrist in an office environment may prefer learning HRVB at home with the support of a remote behavioral health specialist (Gharbo, 2020; Gharbo et. al., 2019).

The current evidence base for HRVB has been developed predominantly with designs that utilize once-weekly high-dose biofeedback protocols. However, there is preliminary evidence indicating that even a brief dose of HRVB improves heart rate regulation, and further, that these skills are internalized and support individuals in regulating physiological aspects of emotional reactivity (Peira et al., 2013). This is promising, as new wearable technologies enable individuals to engage in on-demand low-dose HRVB, which helps remotely build the necessary skills for achieving RSA by syncing breath with heart rate.

Wearable Devices that Measure HRV use Either Electrocardiography (ECG or EKG) or photoplethysmography 
(PPG) technology. ECG technology, considered the gold standard when it comes to measuring heart rate (Singh et al., 2018), uses an electrical voltage signal produced by heart activity to measure HRV (Johns Hopkins Medicine, 2021). Nerve and muscle cells communicate with each other using electrical and chemical signals, which also control heartbeat. This electrical wave throughout the body can be measured on the skin's surface using electrodes with gel, which help conduct readings of the electrical signals. ECG devices measure changes in voltage on different areas of the skin and plot them on a graph. Thus, an electrocardiograph is a visual representation of the electrical signals originating from the heart.

In contrast, PPG technology uses lights and sensors to measure blood volume changes in the cardiovascular system, especially the veins and capillaries under the skin (Allen, 2007). To measure heart rate using PPG technology, an LED shines a constant light onto the skin, some of which is reflected and scattered back into a semiconductor device that converts light into an electrical current, called a photodiode. As the heart beats and sends a pulse throughout the cardiovascular system, the amount of light that reaches the photodiode changes due to pressure changes. PPG technology tracks these changes in light and the time between pulses to determine heart rate (Allen, 2007).

Unlike PPG, which is an indirect method of measuring heart rate, ECG records the heart's electrical activity directly, which makes it more reliably accurate at the millisecond level. ECG technology enables heart rate to be measured accurately on a beat by beat basis, so that meaningful HRV data can be obtained with short-duration measurements. While PPG technology is accurate for measuring HRV in healthy individuals who are at rest, it loses accuracy when physical activity is introduced (Charlot et al., 2009; Schäfer \& Jan Vagedes, 2013). In contrast, the quality of ECG data is more reliable, particularly when movement is introduced. Although ECG technology requires that readings must be conducted close to the heart (e.g. with a patch or chest strap vs. a more familiar watch or ring format), the data is more likely to be detailed, extensive, and accurate.

To examine the feasibility of implementing low dose HRVB using a wearable device in a real world context to improve symptoms of anxiety and increase post-biofeedback HRV, we used an ECG-based HRV wearable device, the Lief Smart Patch, to: (1) Assess compliance to a continuously wearable patch that measures HRV and delivers HRVB, (2) validate efficacy of HRVB delivered through a wearable device in reducing symptoms of anxiety and depression, and (3) validate efficacy of the study's treatment protocol in generating significant increases in HRV pre-post low dose biofeedback session.

\section{Methods}

\section{Participants}

U.S. adults $(18+)$ who wanted help managing their stress were recruited to participate in our study through a Facebook ad (see Appendix A for ad creative and copy) that ran in April 2019. Inclusion criteria included scoring 3 or higher on the Generalized Anxiety Disorder (GAD-2) questionnaire, and scoring 4 or less on the Patient Health Questionnaire (PHQ-2). The primary goal of the study was to assess whether our protocol could help reduce symptoms of anxiety. A score of 3 or higher on the GAD-2 is considered above the clinical threshold for anxiety. However, given that symptoms of anxiety and depression are frequently co-occurring, we also included a measure of depression with the PHQ-2.

The psychometric properties of the PHQ-2 indicate that a score of 5 or 6 indicates a greater than $50 \%$ positive predictive value of major depressive disorder (Kroenke et al., 2003). We excluded those who scored 5 or 6 on the PHQ-2 from the study in order to screen for those who were highly likely to have major depressive disorder, given that it is the most common comorbid disorder involving depression and anxiety (Brown et al., 2001; Gao et al., 2013). We determined that this exclusion criteria adequately balanced the risk of overly limiting our sample pool, given that anxiety and depression disorders often present together, with the study's goal to isolate the intervention's effect on symptoms of anxiety as much as possible.

Other exclusion criteria included having a diagnosed heart condition, taking or planning to take medication for anxiety or depression within the next 3 months, and receiving or planning to receive therapy for anxiety or depression within the next 3 months. In addition, only those with access to an iOS or Android device compatible with the Lief mobile app could participate in the study.

Respondents who fit the study criteria were invited to participate. If they responded yes to the invitation and acknowledged their understanding of and agreement with the electronic consent form, they were enrolled in the study and sent a Lief device. 17 individuals engaged with the study intervention. We define engagement as individuals who began the study intervention, including those who did not complete the study, but excluding those who did not participate due to extenuating medical events unrelated to the study. 14 participants (82\%) completed the study. Three participants partially completed the study, but dropped out before finishing due to: losing the device (1), experiencing technical difficulties (1), and lack of participation with no reason given (1).

Participants were aged 24-47 years (mean $=33, \mathrm{SD}=6)$, with 9 participants identifying as female $(64 \%)$, and 5 
participants identifying as male (36\%). This uneven distribution of gender reflects the statistic that women are twice as likely to be affected by anxiety as men (Anxiety \& Depression Association of America, 2020; Vesga-López et al., 2008). See Table 1 for a breakdown of participant demographics, including baseline anxiety scores.

\section{Study Design}

Our study was approved by the IntegReview Institutional Review Board to investigate how using an electrocardiogram-based wearable device can support individuals in regulating their HRV to reduce symptoms of anxiety. The study took place between July 2019 and September 2019. Participants confirmed their informed consent "I have read and understood the consent form, have no further questions, and wish to participate in the 8-week study," prior to participating. As compensation, participants accrued $\$ 25$ every 2 weeks in the form of an Amazon gift card for a total possible amount of $\$ 100$ upon study completion.

Over the course of 8 weeks, participants were instructed to: Wear the Lief Smart Patch daily for at least $4 \mathrm{~h}$ a day for 40 out of 56 days; complete three guided breathing exercises each day for 40 out of 56 days; interact with a remote stress management coach on the phone for 10 min every 2 weeks; and complete the GAD-2 and PHQ-2 within the Lief mobile app every 2 weeks. Every participant had the same stress management coach. The content covered by the stress management coach applied basic principles from Cognitive Behavioral Therapy (CBT), one of the most widely used and empirically validated psychotherapeutic interventions for anxiety disorders (David et al., 2018; Hofmann et al., 2012), and mindfulness meditation (Hoge et al., 2013) to help manage stress, worry, and anxiety.

Table 1 Participant demographics $(\mathrm{N}=14)$
The structure of the biweekly phone calls were as follows: (Call 1) Introduction to HRV and overview of the 8 -week study, including how to wear the Lief Smart Patch and complete the 3-min HRVB exercise; (Call 2) Identifying and discussing specific triggers for stress and anxiety, and reviewing three different use cases for HRVB exercises (as a routine well-being practice, in preparation for a challenge, and in response to a challenge); (Call 3) Deconstructing stress responses by evaluating situation, physiological response, thoughts, emotions, and behavior; and (Call 4) Managing difficult emotions using the RAIN (Recognize, Allow, Investigate, Non-Identify) mindfulness strategy (Brach, 2020). A study termination call conducted at the end of week 8 provided participants the opportunity to reflect on their experience being in the study, and share feedback.

\section{HRV and HRV Biofeedback Equipment}

We utilized the Lief Smart Patch to collect heart rate, HRV, and accelerometer data through continuous monitoring. The Lief Smart Patch uses ECG technology to measure HRV and deliver HRVB. The Lief Smart Patch was the optimal choice to investigate our research questions, since no other device available to consumers offers a combination of the following characteristics: (1) Ability to wear continuously throughout the day; (2) Ability to deliver HRV biofeedback in the moment; (3) Discreet form factor; and (4) ECG-based measurement of HRV, which is more accurate, especially in ambulatory settings, compared to PPG-based measurement. For example, while another wearable device, the HeartMath ear clip, also delivers HRV biofeedback, it uses PPG rather than ECG technology, and is not discreet enough to wear throughout the day, since it is conspicuously clipped to the ear.

The Lief Smart Patch is worn on the torso underneath clothing and uses vibration feedback to unobtrusively deliver HRVB exercises (see Appendix B for correct placement of the Lief Smart Patch and ECG stickers on the torso). Lief's novel beat-to-beat HRVB protocol (U.S. Patent No. 969,952,8B2) offers real-time, discreet HRVB by giving the user vibration feedback during a specific portion of the RSA. This representation of the RSA amplitude is also mirrored within the accompanying Lief smartphone app, where the user can visualize their heart rate in a constantly updating time series. Rather than creating resonance frequency, the Lief Smart Patch serves as a continual biofeedback mechanism to achieve peak valley differences (i.e., maximum RSA).

The Lief Smart Patch delivers HRVB by initiating vibration feedback when the user's heart rate begins to drop. During this time, the user is cued to exhale by the Lief app. When the user's heart rate begins accelerating again, the algorithm turns the vibration off, and the Lief app cues the
GAD 2 Intake Generalized Anxiety Disorder-2 Intake Scores 
user to inhale. The vibration-led breathing pattern is complemented by a visualization of the user's RSA depicted in the form of a dynamic heart rate graph in the Lief app. This allows the user to observe the amplitude of the RSA created by their breathing pattern during the 3-min HRVB exercise.

The training objective of the Lief Smart Patch's algorithm is to maximally increase RSA by presenting the user with the amplitude of their RSA with each breath, as indicated by the length of the vibration, or visually in the app with the heart rate graph. After the 3-min HRV biofeedback exercise is complete, the user is presented with their session summary data. This summary captures the user's initial HRV, calculated as the mean of the initial $30 \mathrm{~s}$ of their baseline HRV data; the final HRV, calculated as the mean of the final $30 \mathrm{~s}$ of their session; and the change in HRV over the course of the 3-min exercise. Both pre and post HRV measurements are calculated using the RMSSD algorithm.

Data from the Lief Smart Patch was remotely uploaded to a secure cloud server and web-based dashboard that tracks adherence to HRVB exercises, HRVB training efficacy, and self-reported symptoms of anxiety and depression. The Lief Smart Patch's data syncs with participants' smartphones via Bluetooth Low Energy, then is sent over encrypted communication channels to Lief Therapeutics' HIPAA-compliant cloud server. Participants' access to a smart phone was essential, as it enabled users to download the accompanying Lief app, access real-time visual guidance while participating in HRVB, complete the GAD-2 and PHQ-2 every 2 weeks, and build self-awareness by observing their HRV data and RSA in real-time.

\section{Questionnaires}

\section{Generalized Anxiety Disorder (GAD-2)}

The GAD-2 is a brief screening tool that can be used to assess symptoms of anxiety (Kroenke et al., 2007). The GAD-2 consists of the first two questions from the GAD7. Respondents are asked to rate two items on a four-point Likert scale ranging from "not at all" (0) to "nearly every day" (3): "Over the last 2 weeks, how often have you been bothered by the following problems: (1) Feeling nervous, anxious or on edge; (2) Not being able to stop or control worrying." Possible final scores range from 0 to 6 , with a GAD-2 score of $\geq 3$ shown to have a sensitivity of $86 \%$ and a specificity of $83 \%$ for indicating GAD (Kroenke et al., 2007). The cutoff score of $\geq 3$ provided a moderately high balance of sensitivity and specificity in comparison to other cut-off points (Plummer et al., 2016).

\section{Patient Health Questionnaire (PHQ-2)}

The PHQ-2 is a brief screening tool that can be used to assess symptoms of depression (Kroenke et al., 2003; Löwe et al., 2005). Studies have validated the PHQ-2 for individuals aged 12 years and older across multiple population and cultural settings (Manea et al., 2016). The PHQ-2 consists of the first two questions of the Patient Health Questionnaire-9 (PHQ-9), which target core symptoms of depression, depressed mood and anhedonia over the past 2 weeks (American Psychological Association, 2020). Respondents are asked to rate two items on a four-point Likert scale ranging from "not at all" ( (score $=3$ ): "Over the last 2 weeks, how often have you been bothered by the following problems: (1) Little interest or pleasure in doing things; (2) Feeling down, depressed or hopeless." Possible final scores range from 0 to 6 , with a PHQ-2 score $\geq 3$ shown to have a sensitivity of $83 \%$ and a specificity of $92 \%$ for indicating major depression (Kroenke et al., 2003).

\section{Statistical Analysis}

The statistical analysis of data was performed with SPSS v.26. After assessment of assumptions, a one-way ANOVA with repeated measures was used to assess the change in the GAD-2 and PHQ-2 over time. Pre-post change was measured by a paired (two tailed) t-test.

\section{Results}

All participants completed their biweekly stress management coaching sessions on the phone and filled out the biweekly GAD-2 and PHQ-2 questionnaires. The majority of participants (86\%) wore their Lief Smart Patch and completed at least one 3-min HRVB exercise on at least 40 out of 56 days. However, less than half of participants (43\%) completed three or more 3-min HRVB exercises on at least 40 out of 56 days. There were no significant differences in age, gender, intake GAD-2 or PHQ-2 scores between the completers and drop-outs. See Table 2 for descriptive statistics.

The main effect of time was significant $\left(\mathrm{F}_{(5,60)}=14.84\right.$, $\left.p<0.001, \dot{\eta}^{2}=0.55\right)$. Over the course of 8 weeks, participants' mean GAD-2 score dropped three points from 4.6 $(\mathrm{SD}=1.0)$ to $1.7(\mathrm{SD}=0.7)$ out of 6 . The paired t-test for this decrease was $\left(\mathrm{t}_{(13)}=11.0, p<0.001, \eta^{2}=0.90\right)$, with only $14 \%$ of subjects remaining over the clinical threshold of anxiety, defined as a GAD-2 score of three or higher, by the end of the study. The clear and constant drop in mean anxiety levels from week 1 to week 8 of the study is depicted in Fig. 1. 
Table 2 Means and standard deviations for participants' GAD-2 and PHQ-2 scores

\begin{tabular}{lllllll}
\hline & GAD Intake & GAD 1 & GAD 2 & GAD 3 & GAD 4 & GAD 5 \\
\hline Mean & 4.64 & 2.64 & 2.76 & 2.35 & 14 & 1.71 \\
$\mathrm{~N}$ & 14 & 14 & 13 & 14 & 14 & 14 \\
$\mathrm{SD}$ & 1.00 & 1.21 & 1.01 & 0.93 & 0.73 \\
\hline & PHQ Intake & PHQ I & PHQ 2 & PHQ 3 & PHQ 4 & PHQ 5 \\
\hline Mean & 2.93 & 1.86 & 1.62 & 1.54 & 1.08 & 13 \\
$\mathrm{~N}$ & 14 & 14 & 13 & 13 & 1.29 \\
$\mathrm{SD}$ & 1.14 & 1.35 & 1.33 & 1.61 & 1.90 \\
\hline
\end{tabular}

GAD Generalized Anxiety Disorder-2, $P H Q$ Patient Health Questionnaire-2

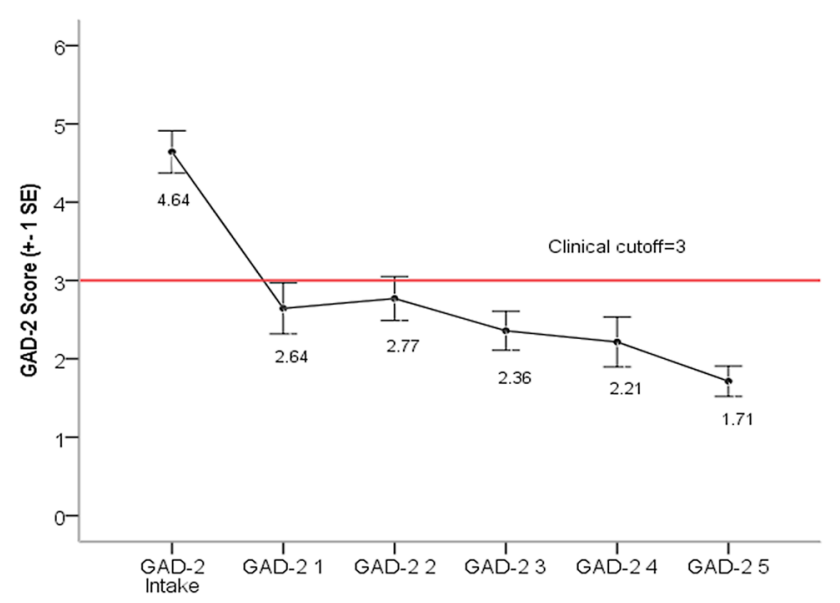

Fig. 1 Mean GAD-2 scores across 6 time points

Similar results were seen for the PHQ-2 depression scale. The main effect for time was significant $\left(\mathrm{F}_{(5,55)}=5.25\right.$, $\left.p<0.001, \dot{\eta}^{2}=0.32\right)$. The paired t-test comparing intake $($ Mean $=2.93, \mathrm{SD}=1.14)$ with time $5($ Mean $=1.29$, $\mathrm{SD}=1.90)$ was $\left(\mathrm{t}_{(13)}=3.54, p<0.01, \eta^{2}=0.49\right)$.

Finally, analysis of over 2700 doses performed during the study showed that study participants on average increased their HRV by + $11.4 \mathrm{~ms}$ for RMSSD during a 3-min dose of Lief's HRVB. No relationship between RMSSD change, total practice minutes, and symptom improvement was seen.

\section{Discussion}

Our study contributes to the emerging evidence supporting self-directed HRVB (Van Der Zwan et al., 2015), and illustrates the potential advantages of utilizing a discreet, continuously wearable HRVB device and remote stress management coach to help manage symptoms of anxiety. Our goals were to: (1) Assess compliance to a continuously wearable patch that measures HRV and delivers HRVB, (2) validate efficacy in reducing symptoms of anxiety, and (3) validate efficacy in generating significant increases in HRV pre-post biofeedback session. Participants' compliance to the study, reduction in symptoms of anxiety over the course of 8 weeks, and increased HRV pre-post dose supports the efficacy of HRVB wearable technology. Furthermore, although it was not a key research question, our results indicate that the study intervention could help reduce symptoms of depression as well.

These findings have positive implications for expanding access to tele-mental health care tools and support for those would benefit from a discreet, flexible option that they can engage with on a daily, as-needed basis. Particularly for individuals who live in a mental health professional shortage area, or are reluctant to pursue more traditional, in-person forms of mental health care due to stigma (Rüsch et al., 2011), mental health care delivered through a HRVB wearable and remote stress management coach may be an attractive alternative option.

The $18 \%$ attrition rate from the initial enrollment, as well as the minority number of participants who completed $100 \%$ of the pilot study requirements highlights the importance of prioritizing engagement when developing a program around an HRVB wearable device. This engagement could come in the form of one-on-one coaching, as well as AI-assisted communication functions that encourage the wearer to maintain consistent adherence. In the future, value based systems in compliance with mental health parity laws may create incentives for attaining skills, like HRVB, which can help improve the overall mental health trajectory. Such incentives will likely motivate patients to adhere to HRVB training, especially if motivated by the positive feedback loop of seeing their objective biomarker data improve (Gharbo, 2020).

\section{Limitations}

The main limitations of this study are the small sample size, and the lack of a control group. As a result, we cannot draw causal inferences about the effects. Greater depth of 
outcome assessment would strengthen the design. A future study should investigate the impact of HRVB and stress management coaching using a randomized controlled trial to compare effect sizes and explore the different outcomes between four participant groups that are demographically representative of the general population: those who receive both HRVB and stress management coaching, those who engage in HRVB only, those who receive stress management coaching only, and a control group that doesn't engage in HRVB or receive stress management coaching. Even though epidemiological data indicates that women are twice as likely to be affected by anxiety than men (Remes et al., 2016), this trend may change as the stigma of mental illness lessens, and men are diagnosed and treated more over time.

Our study examined whether the treatment protocol could help reduce symptoms of anxiety outside a clinical environment. As a result, we utilized the GAD-2 instead of the GAD-7, since we were not aiming to diagnose mental disorders outside of a medical office. Instead, we wanted to show how symptoms of anxiety could be addressed outside the clinic environment, and without the supervision of a medically trained specialist. Future studies could further isolate the effects of this study's treatment protocol on specific diagnosed anxiety disorders. In order to do so, we recommend using the GAD-7 instead of the GAD-2 to establish a more reliable diagnosis of anxiety that offers improved sensitivity and specificity. In addition, such studies could be conducted in collaboration with a mental health clinic to investigate the uses of a remote HRVB program when incorporated with treatment from a medical professional.

\section{Conclusion}

The Mental Health Care Parity Law of 2008 requires insurers to treat physical and behavioral health equally. Unfortunately, it has been largely ignored. An illustrative example is the successful class action lawsuit against Kaiser Permanente (Mental Health Weekly, 2018) for illegally denying patients appropriate mental health care in a timely manner due to consistent shortages in behavioral health care providers. Wearable technology that utilizes remote monitoring and telehealth could help alleviate this ongoing issue.

A discreet wearable device that measures HRV in real time and can deliver HRVB throughout the day could potentially be an effective, affordable adjunct therapy for anxiety when delivered under the guidance of a stress management coach, therapist, psychologist, or other qualified behavioral health care specialist. Notably, our study demonstrated efficacy outside of a specialized mental health center or academic center, which has positive implications for extending care teams outside the clinic.

The findings from our study support the execution of a larger investigation into the topic of how mental health providers may be able to use such technology to benefit their patient populations.

HRVB remote monitoring paired with specialty mental health services has enormous potential, and may be particularly appealing for patients interested in non-pharmacological mental health treatment options. Our preliminary findings indicate that remotely delivered, low-dose biofeedback can be incorporated into daily life to help reduce symptoms of anxiety, outside of a clinical setting. Health and wellness coaches could deploy wearable HRVB devices like the Lief Smart Patch to help their clients, giving them access to efficacious, remotely accessible tools without requiring a medical appointment.

Traditional biofeedback treatment is resource intense, typically limited to a few specialists within mental health. In contrast, HRVB principles can be quickly learned by health care providers, as well as by health and wellness coaches, allowing them to utilize remote HRVB when delivering care and teaching these durable skills to the patient. Wearable devices place mutual responsibility on the patient to allow for ongoing support, while encouraging daily compliance to ensure individuals do not slip through the cracks.

Finally, this study took place prior to the onset of COVID19. Since COVID-19, access to in-person treatment has become more difficult, and many mental health practices that provided once a week HRVB treatment are no longer able to do so. This unusual scenario limiting in person interactions demonstrated that remote technology for HRVB is not just a good option to have available, but also a necessity. Overall, the paradigm featured in our pilot study has the potential to reduce symptoms of anxiety without using pharmacological treatments, and the potential to benefit millions of individuals across the globe.

\section{Appendix A}

See Fig 2. 


\section{Lief Therapeutics}

Sponsored $\cdot 8$

\section{Try our stress management device for free and get $\$ 100$ for your feedback.}

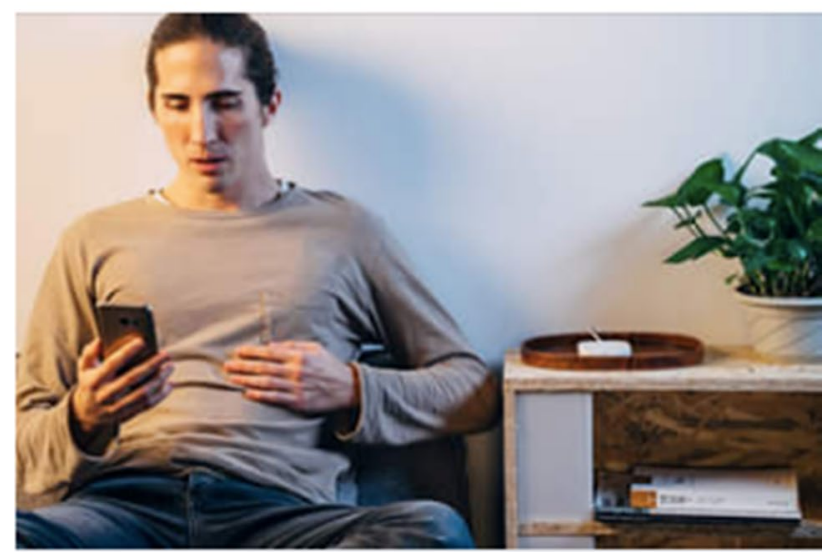

\section{GETLIEF.COM}

Participate in Stress Study

See if you qualify!

\section{d? Like \\ Comment \\ Share}

Fig. 2 Facebook recruitment Ad

\section{Appendix B}

See Fig 3.
Orientation

Battery light on top

Ribbed side to skin

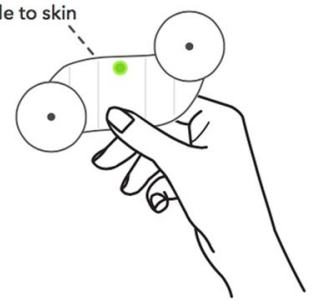

Placement

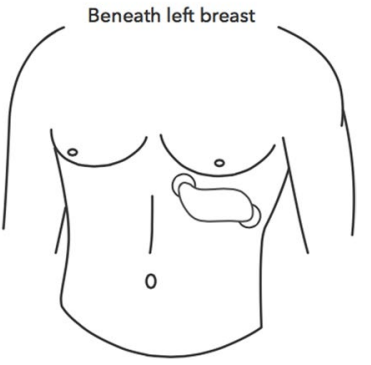

Fig. 3 Instructions for correct placement of the Lief smart patch
Funding Research was supported by Lief Therapeutics, the company that created the Lief Smart Patch, which was utilized in the study to measure participants' heart rate variability and deliver biofeedback training.

Data Availability Data files can be provided upon request. The Lief Smart Patch is available to rent at www.getlief.com/new_order.

Software Availability The free Lief mobile app is available for download for iPhone and Android devices at www.getlief.com/app.

\section{Declarations}

Conflict of interest One author, Adrienne Chung, owns equity in Lief Therapeutics and is their Head of Behavioral Health Communication. The other authors declare that they have no conflict of interest.

Ethical Approval This study was performed in line with the principles of the Declaration of Helsinki. Approval to conduct human subjects research was granted by the IntegReview Institutional Review Board on December 12, 2018 (Protocol Number: 20181127).

\section{References}

Alegría, M., \& O'Malley, I. (2020). Leveraging innovation in behavioral health treatment and its workforce. Harvard Review of Psychiatry, 28(2), 69-71. https://doi.org/10.1097/HRP.0000000000 000250

Allen, J. (2007). Photoplethysmography and its application in clinical physiological measurement. Physiological Measurement, 28(3), R1. https://doi.org/10.1088/0967-3334/28/3/R01

American Psychological Association. (2020). Patient health questionnaire ( $P H Q-9$ \& $P H Q-2)$. American Psychological Association.

American Psychiatric Association. (2020). Update on telehealth restrictions in response to COVID-19. American Psychiatric Association.

Anxiety and Depression Association of America. (2020). Facts \& statistics. Anxiety and Depression Association of America.

Barnett, M. L., \& Huskamp, H. A. (2020). Telemedicine for mental health in the United States: Making progress, still a long way to go. Psychiatric Services, 71(2), 197-198. https://doi.org/10.1176/ appi.ps.201900555

Bishop, T. F., Seirup, J. K., Pincus, H. A., \& Ross, J. S. (2016). Population of US practicing psychiatrists declined, 2003-13, which may help explain poor access to mental health care. Health Affairs (project Hope), 35(7), 1271-1277. https://doi.org/10.1377/hltha ff. 2015.1643

Brach, T. (2020). Radical compassion: Learning to love yourself and your world with the practice of RAIN. Penguin Life.

Brown, T. A., Campbell, L. A., Lehman, C. L., Grisham, J. R., \& Mancill, R. B. (2001). Current and lifetime comorbidity of the DSM-IV anxiety and mood disorders in a large clinical sample. Journal of Abnormal Psychology, 110(4), 585. https://doi.org/10. 1037/0021-843X.110.4.585 
Burch, J.B., Ginsberg, J.P., McLain, A.C., Franco, R., Stokes, S., Susko, K., ... Anderson, A. (2020). Symptom management among cancer survivors: Randomized pilot intervention trial of heart rate variability biofeedback. Applied Psychophysiology and Biofeedback, 45(2), 99-108.

Carter, R. M., Wittchen, H. U., Pfister, H., \& Kessler, R. C. (2001). One-year prevalence of subthreshold and threshold DSM-IV generalized anxiety disorder in a nationally representative sample. Depression and Anxiety, 13(2), 78-88. https://doi.org/10.1002/ da. 1020

Cavanagh, K., Strauss, C., Cicconi, F., Griffiths, N., Wyper, A., \& Jones, F. (2013). A randomised controlled trial of a brief online mindfulness-based intervention. Behaviour Research and Therapy, 51(9), 573-578. https://doi.org/10.1016/j.brat.2013.06.003

Centers for Disease Control and Prevention. (2021). Health and economic costs of chronic diseases. Centers for Disease Control and Prevention.

Chalmers, J. A., Quintana, D. S., Abbott, M. J., \& Kemp, A. H. (2014). Anxiety disorders are associated with reduced heart rate variability: A meta-analysis. Frontiers in Psychiatry, 5, 80. https://doi.org/10.3389/fpsyt.2014.00080

Charlot, K., Cornolo, J., Brugniaux, J. V., Richalet, J. P., \& Pichon, A. (2009). Interchangeability between heart rate and photoplethysmography variabilities during sympathetic stimulations. Physiological Measurement, 30(12), 1357. https://doi.org/10. 1088/0967-3334/30/12/005

Clark, D. M. (1999). Anxiety disorders: Why they persist and how to treat them. Behaviour Research and Therapy, 37, S5-S27.

Czeisler, M., Lane, R., Petrosky, E., Wiley, J., Christensen, A., Njai, R., et al. (2020). Mental health, substance use, and suicidal ideation during the COVID-19 pandemic-United States, June 24-30, 2020. Morbidity and Mortality Weekly Report, 69(32), 1049. https://doi.org/10.15585/mmwr.mm6932a1

David, D., Cristea, I., \& Hofmann, S. G. (2018). Why cognitive behavioral therapy is the current gold standard of psychotherapy. Frontiers in Psychiatry, 9, 4. https://doi.org/10.3389/fpsyt. 2018.00004

Devane, C. L., Chiao, E., Franklin, M., \& Kruep, E. J. (2005). Anxiety disorders in the 21 st century: Status, challenges, opportunities, and comorbidity with depression. The American Journal of Managed Care, 11(12 Suppl), S344-S353.

Ettman, C. K., Abdalla, S. M., Cohen, G. H., Sampson, L., Vivier, P. M., \& Galea, S. (2020). Prevalence of depression symptoms in US adults before and during the COVID-19 pandemic. JAMA Network Open, 3(9), e2019686-e2019686.

Firth, J., Torous, J., Nicholas, J., Carney, R., Rosenbaum, S., \& Sarris, J. (2017). Can smartphone mental health interventions reduce symptoms of anxiety? A meta-analysis of randomized controlled trials. Journal of Affective Disorders, 218, 15-22. https://doi.org/ 10.1016/j.jad.2017.04.046

Fiskum, C. (2019). Psychotherapy beyond all the words: Dyadic expansion, vagal regulation, and biofeedback in psychotherapy. Journal of Psychotherapy Integration, 29(4), 412-425. https://doi.org/10. 1037/int0000174

Gao, K., Wang, Z., Chen, J., Kemp, D. E., Chan, P. K., Conroy, C. M., ... Calabrese, J. R. (2013). Should an assessment of Axis I comorbidity be included in the initial diagnostic assessment of mood disorders? Role of QIDS-16-SR total score in predicting number of Axis I comorbidity. Journal of Affective Disorders, 148(2-3), 256-264. https://doi.org/10.1016/j.jad.2012.12.004

Gevirtz, R. (2013). The promise of heart rate variability biofeedback: Evidence-based applications. Biofeedback, 41(3), 110-120. https://doi.org/10.5298/1081-5937-41.3.01

Gharbo, R.S., Bagherpour, R., \& Lazarus, N. (2019). Untangling chronic pain and hyperarousal with heart rate variability: A case report. Retrieved from https://www.practicalpainmanagement. com/treatments/interventional/untangling-chronic-pain-hyper arousal-heart-rate-variability-case-report

Gharbo, R. S. (2020). Autonomic rehabilitation: Adapting to change. Physical Medicine and Rehabilitation Clinics, 31(4), 633-648.

Gilbert, C., \& Moss, D. (2003). Biofeedback and biological monitoring. In D. Moss, A. McGrady, T. Davies, \& I. Wickramaskera (Eds.), Handbook of mind-body medicine in primary care: Behavioral and physiological tools (pp. 109-122). Sage.

Ginsberg, J., \& Nagpal, M. (2016). Disruption of bradycardia during vigilance: Autonomic cardiac dysregulation is prelude to disinhibition, hyper-arousal, and attention bias in combat veterans with PTSD. In G.E.-B. Fortin (Ed.), A multidimensional approach to post-traumatic stress disorder-From theory to practice. IntechOpen.

Goessl, V. C., Curtiss, J. E., \& Hofmann, S. G. (2017). The effect of heart rate variability biofeedback training on stress and anxiety: A meta-analysis. Psychological Medicine, 47(15), 2578-2586. https://doi.org/10.1017/S0033291717001003

Henriques, G., Keffer, S., Abrahamson, C., \& Horst, S. J. (2011). Exploring the effectiveness of a computer-based heart rate variability biofeedback program in reducing anxiety in college students. Applied Psychophysiology and Biofeedback, 36(2), 101112. https://doi.org/10.1007/s10484-011-9151-4

Hilty, D. M., Ferrer, D. C., Parish, M. B., Johnston, B., Callahan, E. J., \& Yellowlees, P. M. (2013). The effectiveness of telemental health: A 2013 review. Telemedicine Journal and E-Health, 19(6), 444-454. https://doi.org/10.1089/tmj.2013.0075

Hirschfeld, R. M. (2001). The comorbidity of major depression and anxiety disorders: Recognition and management in primary care. Primary Care Companion to the Journal of Clinical Psychiatry, 3(6), 244. https://doi.org/10.4088/pcc.v03n0609

Hofmann, S. G., Sawyer, A. T., Fang, A., \& Asnaani, A. (2012). Emotion dysregulation model of mood and anxiety disorders. Depression and Anxiety, 29(5), 409-416. https://doi.org/10.1002/da. 21888

Hoge, E.A., Bui, E., Marques, L., Metcalf, C.A., Morris, L.K., Robinaugh, D.J., ... Simon, N.M. (2013). Randomized controlled trial of mindfulness meditation for generalized anxiety disorder: Effects on anxiety and stress reactivity. The Journal of Clinical Psychiatry, 74(8), 786. https://doi.org/10.4088/JCP.12m08083

Hunkin, H., King, D. L., \& Zajac, I. T. (2019). Wearable devices as adjuncts in the treatment of anxiety-related symptoms: A narrative review of five device modalities and implications for clinical practice. Clinical Psychology: Science and Practice, 26(3), e12290. https://doi.org/10.1111/cpsp.12290

Jazaieri, H., Goldin, P. R., Werner, K., Ziv, M., \& Gross, J. J. (2012). A randomized trial of mindfulness-based stress reduction versus aerobic exercise for social anxiety disorder. Journal of Clinical Psychology, 68(7), 715-731. https://doi.org/10.1002/jclp.21863

Jelinek, H. F., \& Khandoker, A. H. (2020). Reducing suicidal ideation by biofeedback-guided respiration-Heart rate coherence. Digital Psychiatry, 3(1), 1-11. https://doi.org/10.1080/2575517X.2020. 1732733

Jimenez, D. E., Bartels, S. J., Cardenas, V., \& Alegría, M. (2013). Stigmatizing attitudes toward mental illness among racial/ethnic older adults in primary care. International Journal of Geriatric Psychiatry, 28(10), 1061-1068. https://doi.org/10.1002/gps.3928

Johns Hopkins Medicine. (2021). Electrocardiogram. Johns Hopkins Medicine.

Katon, W. (1996). Panic disorder: Relationship to high medical utilization, unexplained physical symptoms, and medical costs. The Journal of Clinical Psychiatry, 57(Suppl 10), 11-18. https://doi. org/10.1016/0002-9343(92)90130-4

Kim, H. G., Cheon, E. J., Bai, D. S., Lee, Y. H., \& Koo, B. H. (2018) Stress and heart rate variability: A meta-analysis and review of 
the literature. Psychiatry Investigation, 15(3), 235. https://doi.org/ 10.30773/pi.2017.08.17

Kroenke, K., Spitzer, R. L., \& Williams, J. B. (2003). The patient health questionnaire-2: Validity of a two-item depression screener. Medical Care. https://doi.org/10.1097/01.MLR.0000093487. 78664.3C

Kroenke, K., Spitzer, R. L., Williams, J. B., Monahan, P. O., \& Löwe, B. (2007). Anxiety disorders in primary care: Prevalence, impairment, comorbidity, and detection. Annals of Internal Medicine, 146(5), 317-325. https://doi.org/10.7326/0003-4819-146-5-20070 3060-00004

Lee, J., Kim, J. K., \& Wachholtz, A. (2015). The benefit of heart rate variability biofeedback and relaxation training in reducing trait anxiety. The Korean Journal of Health Psychology, 20(2), 391408. https://doi.org/10.17315/kjhp.2015.20.2.002

Lehrer, P. (2013). How does heart rate variability biofeedback work? Resonance, the baroreflex, and other mechanisms. Biofeedback, 41(1), 26-31. https://doi.org/10.5298/1081-5937-41.1.02

Lehrer, P. M., \& Gevirtz, R. (2014). Heart rate variability biofeedback: How and why does it work? Frontiers in Psychology, 5, 756. https://doi.org/10.3389/fpsyg.2014.00756

Lehrer, P., Kaur, K., Sharma, A., Shah, K., Huseby, R., Bhavsar, J., \& Zhang, Y. (2020). Heart rate variability biofeedback improves emotional and physical health and performance: A systematic review and meta analysis. Applied Psychophysiology and Biofeedback. https://doi.org/10.1007/s10484-020-09466-Z

Löwe, B., Kroenke, K., \& Gräfe, K. (2005). Detecting and monitoring depression with a two-item questionnaire (PHQ-2). Journal of Psychosomatic Research, 58(2), 163-171. https://doi.org/10. 1016/j.jpsychores.2004.09.006

Mace, S., Boccanelli, A., \& Dormond, M. (2018). The use of telehealth within behavioral health settings: Utilization, opportunities, and challenges. Behavioral Health Workforce Research Center.

Manea, L., Gilbody, S., Hewitt, C., North, A., Plummer, F., Richardson, R., ... McMillan, D. (2016). Identifying depression with the PHQ-2: A diagnostic meta-analysis. Journal of Affective Disorders, 203, 382-395. https://doi.org/10.1016/j.jad.2016.06.003

Marcus, S. C., Olfson, M., Pincus, H. A., Shear, M. K., \& Zarin, D. A. (1997). Self-reported anxiety, general medical conditions, and disability bed days. American Journal of Psychiatry, 154(12), 1766-1768. https://doi.org/10.1176/ajp.154.12.1766

Mental Health Weekly. (2018). Kaiser Permanente reaches settlement in class action lawsuit. Mental Health Weekly.

Mojtabai, R. (2007). Americans' attitudes toward mental health treatment seeking: 1990-2003. Psychiatric Services, 58(5), 642-651. https://doi.org/10.1176/ps.2007.58.5.642

National Council for Behavioral Health. (2017). The psychiatric shortage: Causes and solutions. National Council for Behavioral Health.

National Institute of Mental Health. (2017). Prevalence of any anxiety disorder among adults. National Institute of Mental Health.

National Institute of Mental Health. (2019a). Prevalence of major depressive episode among adults. National Institute of Mental Health.

National Institute of Mental Health. (2019b). Treatment of major depressive episode among adults. National Institute of Mental Health.

Peira, N., Pourtois, G., \& Fredrikson, M. (2013). Learned cardiac control with heart rate biofeedback transfers to emotional reactions. PLOS ONE, 8(7), e70004. https://doi.org/10.1371/journ al.pone.0070004

Petrowski, K., Wichmann, S., Siepmann, T., Wintermann, G. B., Bornstein, S. R., \& Siepmann, M. (2017). Effects of mental stress induction on heart rate variability in patients with panic disorder. Applied Psychophysiology and Biofeedback, 42(2), 85-94. https://doi.org/10.1007/s10484-016-9346-9

Plummer, F., Manea, L., Trepel, D., \& McMillan, D. (2016). Screening for anxiety disorders with the GAD-7 and GAD-2: A systematic review and diagnostic meta-analysis. General Hospital Psychiatry, 39, 24-31. https://doi.org/10.1016/j.genhosppsych. 2015.11.005

Remes, O., Brayne, C., Van Der Linde, R., \& Lafortune, L. (2016). A systematic review of reviews on the prevalence of anxiety disorders in adult populations. Brain and Behavior, 6(7), e00497. https://doi.org/10.1002/brb3.497

Robinson, W. D., Springer, P. R., Bischoff, R., Geske, J., Backer, E., Olson, M., Jarzynka, K., \& Swinton, J. (2012). Rural experiences with mental illness: Through the eyes of patients and their families. Families, Systems, \& Health, 30(4), 308-321. https:// doi.org/10.1037/a0030171

Rüsch, N., Evans-Lacko, S. E., Henderson, C., Flach, C., \& Thornicroft, G. (2011). Knowledge and attitudes as predictors of intentions to seek help for and disclose a mental illness. Psychiatric Services, 62(6), 675-678. https://doi.org/10.1176/ps. 62.6.pss6206_0675

Salari, N., Hosseinian-Far, A., Jalali, R., Vaisi-Raygani, A., Rasoulpoor, S., Mohammadi, M., ... Khaledi-Paveh, B. (2020). Prevalence of stress, anxiety, depression among the general population during the COVID-19 pandemic: A systematic review and meta-analysis. Globalization and Health, 16(1), 1-11. https:// doi.org/10.1186/s12992-020-00589-w.

Schäfer, A., \& Vagedes, J. (2013). How accurate is pulse rate variability as an estimate of heart rate variability? A review on studies comparing photoplethysmographic technology with an electrocardiogram. International Journal of Cardiology, 166(1), 15-29. https://doi.org/10.1016/j.ijcard.2012.03.119

Schoenberg, P. L., \& David, A. S. (2014). Biofeedback for psychiatric disorders: A systematic review. Applied Psychophysiology and Biofeedback, 39(2), 109-135. https://doi.org/10.1007/ s10484-014-9246-9

Schwartz, M., \& Andrasik, F. (Eds.). (2003). Biofeedback: A practitioner's guide (3rd ed.). Guilford.

Shaffer, F., \& Moss, D. (2006). Biofeedback. In C.-S. Yuan, E. J. Bieber, \& B. A. Bauer (Eds.), Textbook of complementary and alternative medicine (2nd ed., pp. 291-312). Informa Healthcare.

Singh, N., Moneghetti, K. J., Christle, J. W., Hadley, D., Froelicher, V., \& Plews, D. (2018). Heart rate variability: an old metric with new meaning in the era of using mhealth technologies for health and exercise training guidance. Part two: Prognosis and training. Arrhythmia \& Electrophysiology Review, 7(4), 247. https://doi. org/10.15420/aer.2018.30.2

Substance Abuse and Mental Health Services Administration. (2015). Using technology-based therapeutic tools in behavioral health services (SMA-15-4924). Substance Abuse and Mental Health Services Administration.

Totten, A.M., Womack, D.M., Eden, K.B., McDonagh, M.S., Griffin, J.C., Grusing, S., \& Hersh, W.R. (2016). Agency for healthcare research and quality (Technical Briefs, No. 26.) Telehealth: Mapping the evidence for patient outcomes from systematic reviews. Retrieved from https://www.ncbi.nlm.nih.gov/books/ NBK379320/

Twenge, J. M., \& Joiner, T. E. (2020). US Census Bureau-assessed prevalence of anxiety and depressive symptoms in 2019 and during the 2020 COVID-19 pandemic. Depression and Anxiety, 37(10), 954-956. https://doi.org/10.1002/da.23077

U.S. Department of Health and Human Services. (2020). Telehealth: Delivering care safely during COVID-19. U.S. Department of Health and Human Services. 
U.S. Department of Health and Human Services, Health Resources and Services Administration, Bureau of Health Workforce, National Center for Health Workforce Analysis. (2016). National projections of supply and demand for behavioral health practitioners. U.S. Department of Health and Human Services, Health Resources and Services Administration, Bureau of Health Workforce, National Center for Health Workforce Analysis.

Van Der Zwan, J. E., De Vente, W., Huizink, A. C., Bögels, S. M., \& De Bruin, E. I. (2015). Physical activity, mindfulness meditation, or heart rate variability biofeedback for stress reduction: A randomized controlled trial. Applied Psychophysiology and Biofeedback, 40(4), 257-268. https://doi.org/10.1007/ s10484-015-9293-x

Vesga-López, O., Schneier, F. R., Wang, S., Heimberg, R. G., Liu, S. M., Hasin, D. S., \& Blanco, C. (2008). Gender differences in generalized anxiety disorder: Results from the National Epidemiologic Survey on Alcohol and Related Conditions (NESARC). The Journal of Clinical Psychiatry, 69(10), 16061616. https://doi.org/10.4088/JCP.v69n1011

Young, H., \& Benton, D. (2018). Heart-rate variability: A biomarker to study the influence of nutrition on physiological and psychological health? Behavioural Pharmacology, 29, 140-151. https://doi.org/10.1097/FBP.0000000000000383

Publisher's Note Springer Nature remains neutral with regard to jurisdictional claims in published maps and institutional affiliations. 